\section{In vitro culture as an aid to conservation of indigenous ferns: Diplazium proliferum}

\author{
Zubeir M.Golamaully, ${ }^{1}$ \\ Vishwakalyan Bhoyroo, ${ }^{1}$ \\ Nadeem Nazurally, ${ }^{1}$ \\ Vineshwar Gopal'
}

${ }^{1}$ Faculty of Agriculture, University of

Mauritius, Reduit; ${ }^{2}$ National Parks and

Conservation Services, Reduit, Mauritius

\begin{abstract}
With the ever growing population and economic needs of Mauritius, the flora of Mauritius has never been in more danger and one group of vascular plants is even more in peril; ferns. Diplazium proliferum is indigenous to the Mascarene region and is considered as a rare species in Mauritius. The need to develop a tested in vitro propagation protocol is a must to protect the biodiversity of Mauritius. This experiment was geared towards the establishment of a proper sterilization technique and the effect of 6-benzylaminopurine (BAP) and light on in vitro culture of this fern. Sterilization with $0.05 \%$ Mercuric chloride was effective to eliminate fungal contamination and allow germination of spores. Culture media supplemented with BAP did not significantly increase growth rate of both gametophytes and sporophytes of $D$. proliferum. Present results suggest efficient sterilization methods to be a crucial stage for successful in vitro regeneration of ferns. The established protocol will be used as an optimized baseline protocol for the propagation of other indigenous ferns.
\end{abstract}

\section{Introduction}

Ferns are referred to as the disaster taxa since during intervals of environmental stress; they are the early pioneers to a newly stripped landscape. ${ }^{1,2}$ The ground work established on the status of ferns in Mauritius began with the project Flore des Mascareignes. The initiative was to describe all the higher plants species and ferns in the Mascarenes. While being near its completion, it has revealed more than 100 new species. Before its colonization, the island of Mauritius was entirely covered with forest. Today, not only about $5 \%$ of the native forest is remaining which sums up to about $100 \mathrm{~km}^{2}$, but it is also threatened by alien invasive species. $40 \%$ of the native flora has been categorized as threatened and about 39 plant species has been confirmed as extinct.

There are four main stages of the growth of a fern from its spore i) the sporophyte or fern plant, ii) nonsexual spores produced as a result of reduction division, iii) gametophyte or thallus plant, iv) oospores produced as the result of the conjugation of egg and sperm. When a spore germinates, it produces a small filament (protonema). Development of the gametophyte occurs through two stages. In the first stage, the plant shape is a linear aggregate and in the second stage, it is a solid aggregate. The thallus is hermaphrodite and hence can develop several spermaries and ovaries on the lower region. Fertilization occurs when a spermatozoid passes down the neck to the egg to conjugate with it. The developing embryo is completely parasitic to the parent plant which provides it with nutrients. Finally the embryo breaks free from the enlarged venter of the archegonium to grow roots in the ground below while a first leaf develops towards the light shifting from a parasitic stage to an independent one. ${ }^{3}$ Gametophytes that germinate from the single celled spore are the source from which a homosporous fern's bisexual gametophyte are mitotically derived. The self-fertilization of a sperm and egg of a single gametophyte that are genetically similar will yield a sporophyte that is instantly homozygous at all loci on its homologous chromosomes. ${ }^{4}$ In situ conservation of ferns and their allies in their natural habitats should always be the main aim. However, this is not always possible; hence, additional methods are required to support and complete the in situ methods which will increase the probability of survival of the individual species. ${ }^{5}$ Several reports of in vitro propagation of spores have shown success in their cultivation but have indicated a considerable loss of spores during the sterilization process, aseptic sowing or due to contamination which have led to the development of different spore sterilization and sowing methods. ${ }^{6}$ In vitro germination of spores and following prothallus development and subsequent sporophyte growth can bypass some of the difficulties linked with conventional methods. These difficulties include: low germination rates, contamination with prothalli from invasive species and the growth of too few sporophytes. Successful in vitro germination of rare species are Cibotium schiedei from Central America and Angiopteris boivinii from the Seychelles. ${ }^{7}$

Spores viability can vary drastically among pteridophytes; from a few days to a few years. It has been understood that the genetic makeup and a few physiological characteristics of spores need to be reviewed to understand the spores' viability variations. ${ }^{8}$ Factors such as spore type, chlorophyllous and non-chlorophyllous or taxonomic region can affect the viabil-
Correspondence: Vishwakalyan Bhoyroo, Department of Agricultural and Food Sciences, Faculty of Agriculture, University of Mauritius, Reduit, Mauritius.

Tel.: +230.797.8038; Fax: +230.403 .7830 .

E-mail:v.bhoyroo@uom.ac.mu

Key words: Ferns; in vitro culture; Diplazium proliferum; conservation.

Acknowledgements: this project was supported by the National parks and Conservation Services (Mauritius). Laboratory facilities were provided by the Faulty of Agriculture and the whole project was funded by the University of Mauritius. We also extend our gratitude to Mr Pynee of the MSIRI for providing accession numbers.

Contributions: the authors contributed equally.

Conflict of interests: the authors declare no potential conflict of interest.

Funding: this project was funded by the University of Mauritius and supported by the National Parks and Conservation Services.

Received for publication: 16 May 2015. Accepted for publication: 24 July 2015.

This work is licensed under a Creative Commons Attribution NonCommercial 3.0 License (CC BYNC 3.0).

(O) Copyright Z. M.Golamaully et al., 2015 Licensee PAGEPress srl, Italy

International Journal of Plant Biology 2015; 6:6020 doi:10.4081/pb.2015.6020

ity of spores The high rate of respiration or inability to recover photosynthetic properties after desiccation in green spores has been proposed as the cause of quick decrease of viability. Lloyd and Klekowski (1970) have compared the viability of green spores (chlorophyllous) and non-green spores (non-chlorophyllous) and noticed that green spores germinate faster than non-green spores; however, this property to germinate is lost quickly over time. ${ }^{9}$ Furthermore, their data has shown that under laboratory conditions, green spores have short life span (48 days) while the non-green spores live on to about 2.8 years. It has also been suggested that the short viability of green spores of Equisetum hyemale L. may be due to the loss of its photosynthetic ability when the spores are rehydrated following desiccation. While the factors influencing viability of spores are known, little is known about those affecting the spores under herbarium conditions. ${ }^{5}$

$D$. proliferum is a rare fern restricted to few forest areas in Mauritius and is listed among the top priorities for immediate propagation and conservation by The Nation Parks and Conservation Services. This study aims to 
develop a tissue culture protocol for the propagation of this wild fern, including defining key stages for successful micropropagation such as sterilization steps and culture condition.

\section{Materials and Methods}

After acquisition of proper permits to collect samples, mature leaflets of Diplazium proliferum (accession number: MAU 26445) were kindly provided by the National Parks and Conservation. Mature leaflets (Figure 1A) were allowed to air dry prior to collection of spores. Dried leaves were scrapped with a scalpel on a sheet of paper and the spores that fell on the paper were transferred in a corning tube. Spore surface sterilization were carried in the 3 following methods i) treatment 1: $2 \%$ Sodium Hypochlorite, ${ }^{10}$ ii) treatment 2: $2 \%$ Sodium Hypochlorite and $1 \mathrm{~g} / \mathrm{L}$ Nystatin (fungicide) and iii) treatment $3: 1 \%, 0.1 \%$ and $0.05 \%$ Mercuric chloride $\left(\mathrm{HgCl}_{2}\right)$. Basal Murashige and Skoog's (1962) media was used as control $(\mathrm{n} \geq 50)$ and $1 \mathrm{mg} / \mathrm{L} \quad 6$ Benzylaminopurine $(n \geq 50)$ was tried as a growth promoter for spore germination. ${ }^{11}$ Cultured pots were partly kept in total darkness in an incubator and partly in light condition for daily 16 hours photoperiod. All experiments were done in aseptic conditions. Following the growth of some sporophytes, the media became insufficient and sub-culturing had to be done. Sporophytes were removed from the media and cut in pieces and inoculated in fresh media (with and without BAP).

Growth rates were measured as surface areas of growing sporophytes using digital photography. Photographs of growing explants were taken with a scale stuck to the wall of culture pots. Surface areas were calculated using Scion Image software. The values obtained were calculated as mean and significant growth differences between treatments were calculated following Mann-Whitney $U$ test in SPSS 16.0. Mann-Whiney U test was used since the samples obtained in the experiments were not normally distributed and had unequal sample size due to different contamination levels. $^{12}$

\section{Results}

First response occurred after 35 days in light (Figure 1B). Different growth phases from the inoculation of spores to the development of green gametophyte stage and consequently to the sporophyte stage with appearance of first leaflets were observed (Figure 2A-C). Slurry like gametophyte usually dries up and then small callus started to form (Figure 2D-F).
Sterilization with $2 \%$ sodium hypochlorite gave too much contamination following the inoculation and the spores did not germinate at all. Supplementing with a fungicide, Nystatin, also gave high contamination levels. Treatments with $1 \%$ and $0.1 \%$ mercuric chloride resulted in minimal contamination levels but no germination were observed at all. Mercuric chloride at $0.05 \%$ was efficient for with this treatment (Figure 3A).

Germination occurred irrespective of presence or the absence of BAP. Furthermore, the rate of development of the gametophytes and sporophytes was not significantly faster (Mann-Whitney U, P>0.05) though higher rates were observed with BAP (Figure 3B,C). The growth rate over the 4 month appeared to sterilization and the germination rate was $51 \%$

be favored by the presence of BAP in the culture media with greater rate of development beginning from the $2^{\text {nd }}$ month. From month 3 , the drop in surface area was due to the subculturing carried out. However quickly after in month 4 , the growth rate spurred back.

\section{Discussion and Conclusions}

Although sodium hypochlorite was reported as an effective and non damaging surface sterilizer, it was not effective in this experiment and extensive contamination was obtained. Interestingly it was reported that surface sterilization of Cyathea gigantea spores at 30\% sodium hypochlorite proved to be effective. ${ }^{13}$

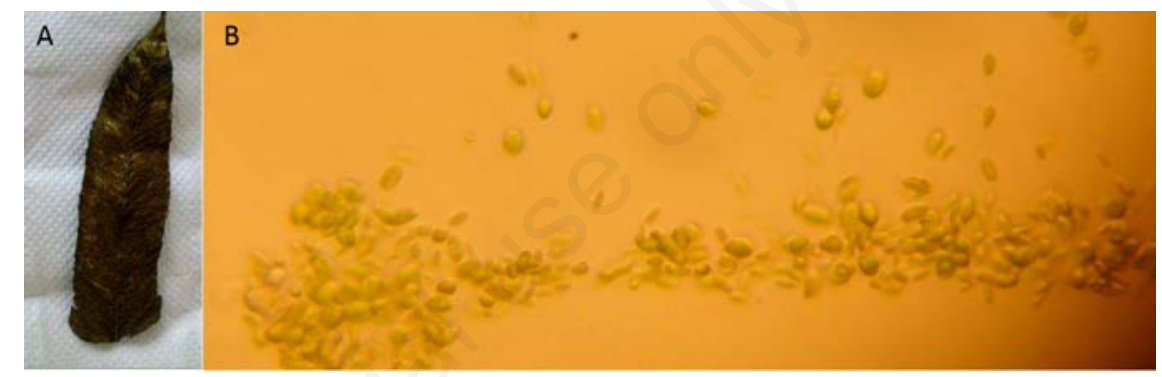

Figure 1. Diplazium proliferum: A) mature leaves with Sporangia clusters on back of leaves; B) cells at gametophytic stage viewed under compound microscope.

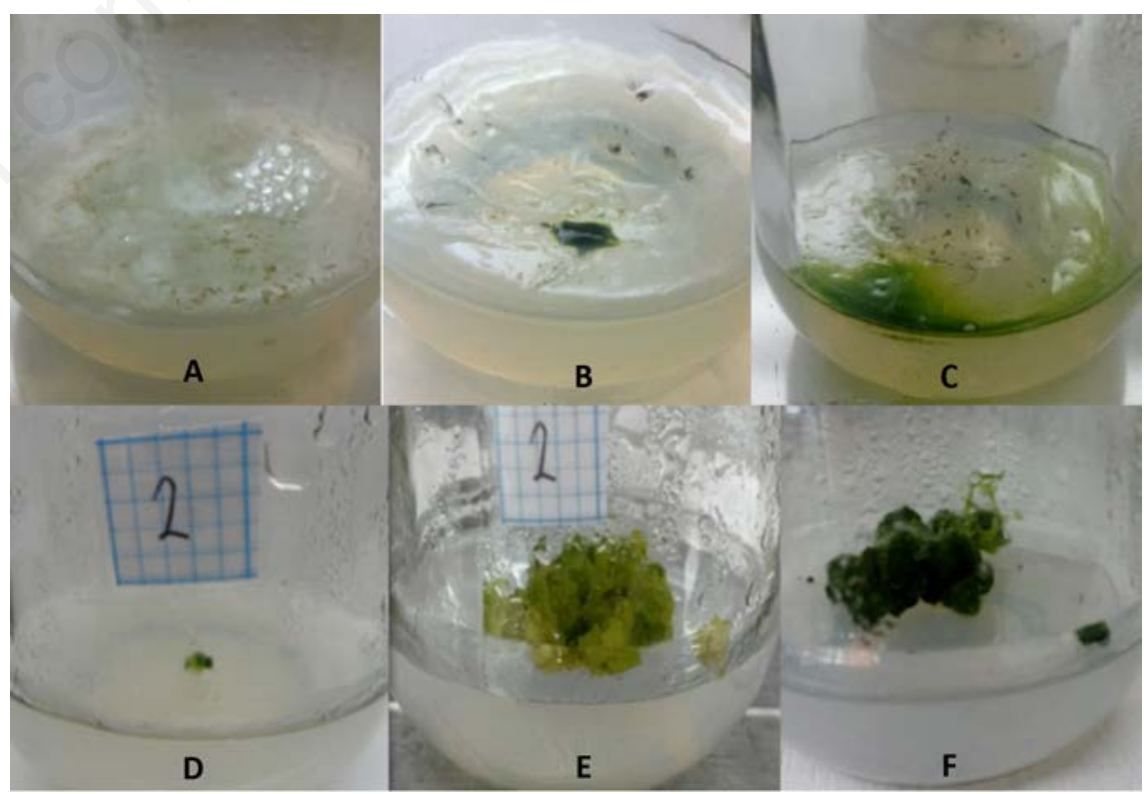

Figure 2. A-C) The development of spores to gametophytic stage: A) inoculated spores, B) initial growth of gametophytes, C) slurry of gametophytes. D-F) Development of sporophyte to small plantlets shoots: D) initial subcultured tissue, E) increase in size of sporophyte, F) appearance of first leaves. 
With lower concentration, the sterilization was not successful and when used above $30 \%$ the mortality rate was too high. However, for the sterilisation of spores of Diplazium proliferum, $2 \%$ sodium hypochlorite proved to be inefficient against fungal contaminants. Since most of the contamination present appeared to be fungal, a fungicide (Nystatin) was used in addition to $2 \%$ sodium hypochlorite. ${ }^{14}$ However, this also proved to be inadequate since, fungal contamination persisted. A stronger sterilizing agent had to be used, since, no success had been made in neutralizing the unwanted microorganisms that were proving to be a major cause preventing germination. Since, mercuric chloride has shown
A

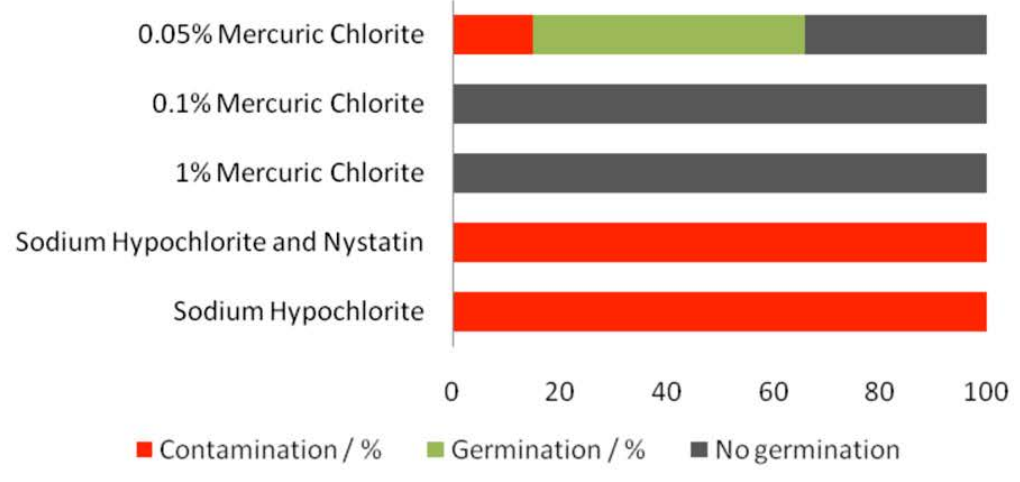

B

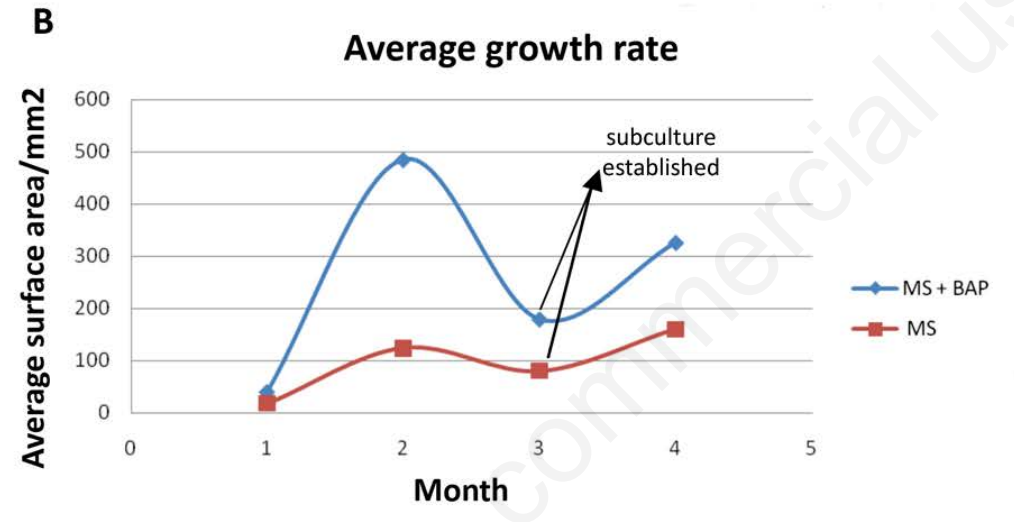

C
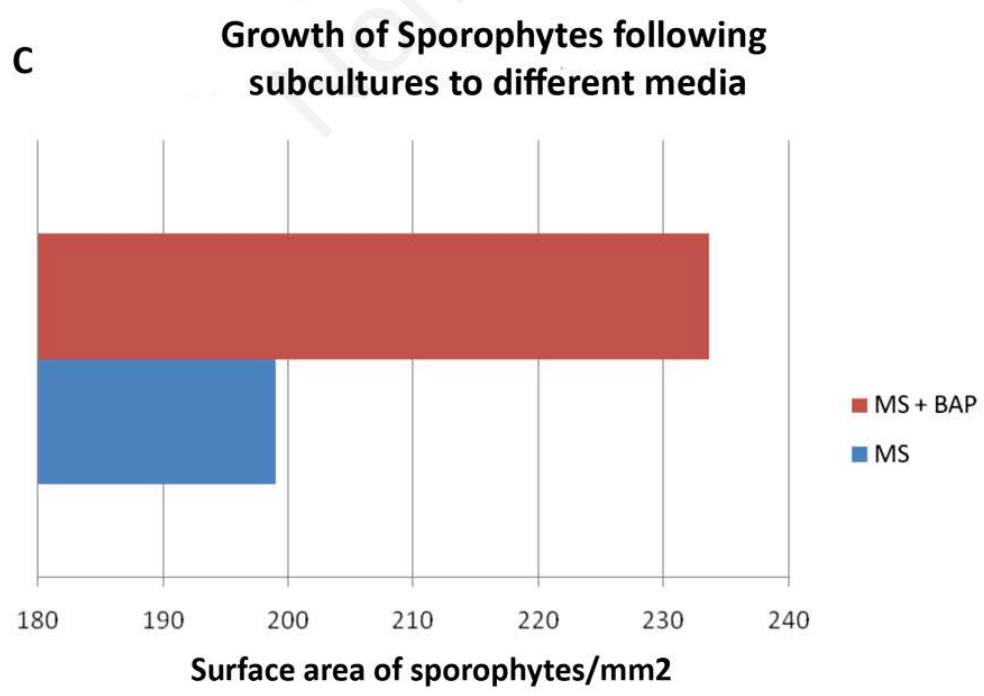

Figure 3. Efficiency of sterilization treatments (A), average growth rate from spores inoculated (B) and difference in growth of sporophytes after subculturing (C). itself as a very strong decontamination agent, its use made sense. Several reports showed promising results from the use of mercuric chloride. ${ }^{15-17}$ The use of $1 \%$ and $0.1 \%$ was too strong and rendered the spores infertile however, the use of $0.05 \%$ produced viable sterile spores which led to the formation of gametophytes for Diplazium proliferum.

The germination of spores varies depending on their taxonomy. ${ }^{9}$ For spores to germinate, it must first have achieved maturation. This is difficult to determine specially in the understudied Diplazium family. Several factors may affect spore maturation such as the causeeffect relationship whereby dry weather, high temperature and low humidity favored release of mature spores. ${ }^{18}$ Once spores had germinated it would give rise to a gametophyte which produces a sporophyte if successful fertilization occurs. This series of events is dependent on many factors. While germination of a spore will most probably give rise to a prothallus, it will not necessarily guarantee a sporophyte. This can be explained by the fact that all the gametophytes that emerged were hermaphroditic that is without the presence of an egg and a sperm simultaneously, no sporophyte could emerge regardless of other factors. Presence of lethal or semi lethal genes following fusing of an egg and sperm will ensure that the sporophyte dies. The genotype of this gametophyte is deadly in the homozygous state. This phenomenon and found that in seven population of Osmunda regalis from which he obtained 109 sporophytes, all possessed at least 1 form of lethal gene in heterozygous condition. ${ }^{19}$ Though BAP was reported to positively affect the growth of ferns in vitro, ${ }^{20}$ it did not significantly increase the growth rates for Diplazium proliferum. However, it should be noted that the non-uniformity in the data obtained was mainly due to the problem of contamination. This study proved that sterilization of spores for in vitro culture is a very crucial stage for successful in vitro propagation of ferns from spores.

\section{References}

1. Tschudy RH, Pillmore CL, Orth CJ, et al. Disruption of the terrestrial plant ecosystem at the Cretaceous-Tertiary boundary, Western Interior. Science 1984;225:1030-2.

2. McElwain JC, Punyasena S. Mass extinction events and the plant fossil record. Trends Ecol Evol 2007;22:548-57.

3. Schaffner JH. A life cycle of a Homosporous pteridophyte. Ohio J Sci 1906;6:485-8.

4. Nakazato T, Jung MK, Housworth EA, et al. Genetic map-based analysis of genome structure in the homosporous fern 
Ceratopteris richardii. Genetics 2006;173:1585-97.

5. Magrini S, Olmati C, Onofri S, Scoppola A. Recovery of viable germplasm from herbarium specimens of Osmundaregalis L. Am Fern J 2010;100:159-60.

6. Hua W, Chen PT, Yuan LP, Chen LQ. An efficient method for surface sterilization and sowing Fern spores in vitro. Am Fern $\mathrm{J}$ 2009;99:226-30.

7. Fay MF. Conservation of rare and endangered plants using in vitro methods. In Vitro Cell Dev Biol 1992;28:1-4.

8. Camloh M. Spore age and sterilization affects germination and early gametophyte development of Platyceriumbifurcatum. Am Fern J 1999;89:124-32.

9. Lloyd RM, Klekowski EJ Jr. Spore germination and viability in Pteridophyta: evolutionary significance of chlorophyllous spores. Biotropica 1970;2:129-37.

10. Knauss JF. A partial tissue culture method of pathogen-free propagation of selected ferns from spores. Proc Florida State Horticult Soc 1976;8:848-52.

11. Murashige T, Skoog F. A revised medium for rapid growth and bio-assays with tobacco tissue cultures. Physiol Plant 1962;15:473-97.

12. Nachar N. The Mann Whitney U: a test for assessing whether two independent samples come from the same distribution. Tutorials Quant Methods Psychol 2008;4:13 20.

13. Das B, Tania P, Kishori GA, et al. Evaluation of antioxidant potential \&amp; quantification of polyphenols of Diplazium esculentum Retz. with emphasis on its HPTLC chromatography. J Pharm Res 2013;6:93-100.

14. Brumback WE. Raising the climbing fern from spores. Arnoldia 1985;45:27-9.

15. Danso KE, Azu E, Elegba W, et al. Effective decontamination and subsequent plantlet regeneration of sugarcane (Sacchrum officinarum L.) in vitro. Int $\mathrm{J}$ Integr Biol
2011;11:90-6.

16. Puchooa D. In vitro regeneration of lychee (Litchi chinensis Sonn.). Afr J Biotechnol 2004;3:576-84.

17. Badoni A, Chauhan JS. In vitro sterilization protocol for micropropagation of solanum tuberosum cv. Kufri Himalini. Academia Arena 2010;2:24-7.

18. Arosa ML, Quintanilla LG, Ramos JA. Spore maturation and release of two evergreen macaronesian ferns, Culcita macrocarpa and Woodwardia radicans, along an altitudinal gradient. Am Fern J 2009;99: 260-72.

19. Klekowski EJ Jr. Populational and genetic studies of a homosporous fern-osmunda regalis. Am J Botany 1970;57:1122-38.

20. Bonomo MC, Martínez OG, Tanco ME, et al. Spores germination and gametophytes of Alsophila odonelliana (Cyatheaceae) in different sterile media. YTON 2013;82:11926. 